Research Article

\title{
Elastic Analysis for Rotating Functionally Graded Annular Disk with Exponentially-Varying Profile and Properties
}

\author{
Wen-Feng Lin (i) \\ College of Mechanical and Automotive Engineering, Zhaoqing University, Zhaoqing, Guangdong, China \\ Correspondence should be addressed to Wen-Feng Lin; 3130637143@qq.com
}

Received 8 August 2020; Revised 9 October 2020; Accepted 29 October 2020; Published 16 November 2020

Academic Editor: Daniela Addessi

Copyright (C) 2020 Wen-Feng Lin. This is an open access article distributed under the Creative Commons Attribution License, which permits unrestricted use, distribution, and reproduction in any medium, provided the original work is properly cited.

\begin{abstract}
Functionally graded materials have been widely used in engineering and human health applications. The issues about mechanical behavior of functionally graded material have received considerable attention. However, because of the complexity of material property, geometric profile, and mechanical load, there is still lack of proper analytic solutions about deformation and stress in many articles. The principal goal of this research is to study the effect of mechanical load on deformation and stress in rotating thin-walled functionally gradient material annular disk with exponentially-varying profile and properties. The inner and outer surfaces of annular disk are subjected to different pressures simultaneously. For this purpose, the infinitesimal theory of elasticity and axisymmetric plane stress assumptions has been proposed to formulate the governing equation. The governing equation is a generalized confluent hypergeometric differential equation, based on Whittaker's functions; this is the first time that closed-form solutions of mechanical behaviors are revealed about proposed functionally gradient material model. Besides, another four boundary conditions are also discussed, i.e., the inner and outer surfaces of the annular disk are considered to be the combinations of free and clamped conditions. Numeric examples of two different functionally graded material properties are given to demonstrate displacement and stress solutions. Moreover, uniform disks made of homogeneous material under different boundary conditions are investigated, which are special cases of the proposed rotating functionally gradient material disks. Finally, some conclusions are made at the end of the present paper.
\end{abstract}

\section{Introduction}

Annulus disks attached on a shaft to be a rotating machinery element are widely used in shrink fits, gears, flywheels, disc brakes, ship propellers, and many other engineering applications. In tradition, the disk is made of homogeneous material and the thickness is uniform along the radial direction. The analyses of uniform disks made of homogeneous material have paid much attention in a study by Timoshenko and Goodier [1]. Further, many researchers investigate the mechanical behavior of homogeneous and isotropic material with nonuniform thickness. Nayak and Saha [2] investigated the influence of thermo-mechanical loading on stress and deformation in a rotating disk with varying thicknesses. However, their results were not analytic solutions and were accomplished by numerical method to achieve estimated solutions. Yildirim [3] proposed closed- form formulas to discuss the mechanical behaviors for hyperbolic disks made of conventional materials under different boundary conditions, but the proposed material properties such as Young's modulus and density are constant.

In some environments, the homogeneous and isotropic disk cannot endure high strength. Functionally gradient material (FGM) may endure simultaneously high strength and high temperature which have attracted many attentions. Functionally gradient material is composed of two or more material ingredients, the different mechanical properties vary continuously from one surface to the other. Durodola and Attia [4] discussed displacement and stress of FGM with uniform thickness. They explored displacement and stress of the rotating hollow and solid disks by direct integration method and finite element method. However, as the integration method was marched forward step by step from the 
inner surface to the outside surface, the results are not closed-form solutions. Shi et al. [5] studied a uniform thickness FGM hollow cylinder with continuously graded properties, which is subjected to uniform pressures on the inner and outer surfaces. Young's modulus is changing linearly in radial direction. They found the solutions of displacement and stress by converting the FGM hollow cylinder to the $N$-layered homogeneous elastic hollow cylinder. Therefore, the results are not closed-form solutions, either. You et al. [6] developed analytical solutions to determine deformation and stresses of FGM annular disk, which is subjected to external and/or internal pressure on boundary surfaces. Young's modulus and Poisson's ratio are both represented as linear functions of radius, but the considered annular disk thickness is uniform. Dai and Dai [7] studied the displacement and stress distribution in a rotating functionally graded material disk with variable angular speeds. In their research, the considered FGM circular disk thickness was uniform, so they got mechanical behaviors of the rotating FGM circular disk in numerical solutions. Nejad et al. [8] used hypergeometric function defined by Abramowitz and Stegun [9] to present elastic analysis of FGM thick-walled hollow cylindrical pressure vessel in plane strain condition. The vessel was subjected to internal and external pressure at the inner and outer surfaces, respectively. However, the only discussed property of FGM was elasticity modulus, and the thickness was uniform. Gharibi et al. [10] used the Frobenius series method to present the elastic analysis of rotating FGM thick hollow cylindrical pressure vessel. Young's modulus and Poisson's ratio were both assumed as exponentially-varying functions along the radial direction presented by Sadd [11]. But the hollow cylindrical vessel thickness was uniform and the vessel density was considered as a constant. Thawait [12] analyzed hollow rotating cylindrical pressure vessels subjected to internal pressure; functional gradation of the FGM properties was done by exponential law. But the vessel thickness was uniform, and the deformation and stress solutions are numerical solutions. The related analyses on rotating functionally graded material disks with uniform thickness have been performed by Eraslan and Akis [13], Zenkour [14], Akbari and Ghanbari [15], Strashnov et al. [16], Salehi Kolahi et al. [17], Yildirim et al. [18], etc.

A few researchers concentrated their works on the analysis of rotating functionally graded material with nonuniform thickness. You et al. [19] analyzed the deformation and stress in rotating solid and/or annular disks with uniform and/or variable thickness at constant and/or variable density. Runge-Kutta algorithm was introduced to solve the governing equation. But Young's modulus of the considered material was constant and the found deformation and stress distributions were not analytic solutions. Bayat et al. [20] used a semianalytical method to analyze rotating gear wheels made of functionally graded material. The material properties and thickness profile were assumed to be represented by two power-law distributions. In their research, they used the semianalytical method to divide radial domain into some virtual subdomains. In each subdomain, the mechanical property was assumed to be constant, so the results are not closed-form solutions. Jalali and Shahriari [21] investigated elastic stress analysis of the rotating variable thickness FGM annular disk. The variation of thickness, Young's modulus, and density of the disk were assumed to be graded by power-law function in the radial direction. Stress and deformation of the rotating FGM annular disk were obtained using the numerical finite difference method. Yildirim and Tutuncu [22] dealt with instability analysis of FGM rotating disks of variable thickness. Instability was determined at critical angular velocity which caused unbounded displacement and stresses. Analytical solutions could be found only for certain material grading functions and uniform thickness profiles. Thawait et al. [23] developed a finite element method to make elastic analysis on concave thickness functionally graded rotating disks. Three types of material models were considered. But the deformation and stress distributions were not analytic and results were only evaluated under clamped-free boundary condition. The related analyses on rotating functionally graded material disks with nonuniform thickness have been performed by Bayat et al. [24], Liu and Chien [25], Zheng et al. [26], Yildirim [27], Shahriari and Safari [28], Gao et al. [29], Yu et al. [30], etc.

Although different material properties of FGM annular disks with uniform and/or variable thickness are extensively studied, to the best of the author's knowledge, the content of this study has not been conducted in the literature. To this aim, this study is to derive analytical solutions of the deformation and stresses for rotating FGM annular disk with exponentiallyvarying thickness and properties. Mechanical loads apply on inner and outer surfaces of the annular disk simultaneously.

In the following, the governing equation of radial displacement will be derived from the equilibrium equation for the thin-walled FGM annular disk in radial direction. The governing equation is a generalized confluent hypergeometric differential equation and the solution is represented by Whittaker's functions in a study by Abramowitz and Stegun [9]. The coefficients in the closed-form solutions can be found by boundary conditions at inner and outer surfaces. Radial displacement and radial and circumferential stress solutions were also obtained under four boundary conditions. The four boundary conditions are as follows: both edges in inner and outer surfaces are free of constraints (circular annulus); both edges are clamped (disk mounted on a rotating shaft at inner surface and cased at outer surface); inner edge is clamped, outer edge is free of constraints (disk mounted on a rotating shaft at inner surface); and inner edge is free of constraints, outer edge is clamped (disk cased at the outer surface). Numerical examples are given to demonstrate the validity of the proposed solutions. Finally, the summaries of the most important achievements are proposed and the future works are also presented at the end of the conclusions.

\section{Theory and Formula}

In this article, we consider a thin-walled functionally graded material annular disk with inner radius $a$ and outer radius $b$. The thickness at inner and outer surfaces is $h_{i}$ and $h_{o}$, 
respectively, as shown in Figure 1. The disk has variable thickness $h(r)$ and its profile is assumed to vary radially in an exponential form defined by

$$
h(r)=h_{i} e^{(-k(r-a) / b)},
$$

where $r$ is the radial coordinate and index $k$ is the profile parameter given by

$$
k=\frac{b}{b-a} \ln \left(\frac{h_{i}}{h_{o}}\right) .
$$

The annular disk rotates about its axis at constant angular velocity $\omega$. At inner and outer surfaces, they are subjected to internal and external pressures $P_{i}$ and $P_{o}$, respectively.

Poisson's ratio $v$ of the considered functionally graded material is assumed to be constant. Young's modulus at inner and outer surfaces is $E_{i}$ and $E_{o}$, density at inner and outer surfaces is $\rho_{i}$ and $\rho_{o}$, respectively. The nonhomogeneous compositions of the considered functionally graded material are assumed to be function of the volume fraction of the constituent materials. The mixture rule of Young's modulus and density in radial direction may vary radially according to the following exponentially-varying functions:

$$
\begin{aligned}
& E(r)=E_{i} e^{(-m(r-a) / b)}, \\
& \rho(r)=\rho_{i} e^{(-n(r-a) / b)},
\end{aligned}
$$

where indexes $m$ and $n$ are the material parameters given by

$$
\begin{gathered}
m=\frac{b}{b-a} \ln \left(\frac{E_{i}}{E_{o}}\right), \\
n=\frac{b}{b-a} \ln \left(\frac{\rho_{i}}{\rho_{o}}\right) .
\end{gathered}
$$

The thickness of the FGM thin-walled disk is assumed to be sufficiently small compared to its diameter. The Kirchhoff strain-displacement relations are

$$
\begin{aligned}
\varepsilon_{r}(r) & =\frac{\mathrm{d} u(r)}{\mathrm{d} r}, \\
\varepsilon_{\theta} & =\frac{u(r)}{r},
\end{aligned}
$$

where $u(r)$ is the radial displacement and $\varepsilon_{r}(r)$ and $\varepsilon_{\theta}(r)$ are the strains in the radial and circumferential directions. In the condition of axisymmetric plane stress assumptions, stressstrain relations are given by

$$
\begin{aligned}
& \sigma_{r}(r)=\frac{E(r)}{1-\nu^{2}}\left[\varepsilon_{r}(r)+\nu \varepsilon_{\theta}(r)\right], \\
& \sigma_{\theta}(r)=\frac{E(r)}{1-\nu^{2}}\left[\varepsilon_{\theta}(r)+\nu \varepsilon_{r}(r)\right],
\end{aligned}
$$

where $\sigma_{r}(r)$ and $\sigma_{\theta}(r)$ are stresses in radial and circumferential directions.
In the absence of body force, the equation of equilibrium for the FGM annular disk in radial direction can be represented as

$$
\frac{\mathrm{d}}{\mathrm{d} r}\left[h(r) r \sigma_{r}(r)\right]-h(r) \sigma_{\theta}(r)+h(r) \rho(r) \omega^{2} r^{2}=0 .
$$

Using equations (1)-(9), let $\beta=((k+m) / b)$, $\Omega=b \sqrt{\left(\rho_{i}\left(1-v^{2}\right) / E_{i}\right)}, R=(r / b), U=(u / b)$, and $\bar{a}=(a / b)$; the governing equation of radial displacement becomes

$$
R^{2} \frac{\mathrm{d}^{2} U}{\mathrm{~d} R^{2}}+R(1-\beta b R) \frac{\mathrm{d} U}{\mathrm{~d} R}-(\nu \beta b R+1) U=-\Omega^{2} R^{3} e^{-(n-m)(R-\bar{a})} \omega^{2} .
$$

The above equation is a generalized confluent hypergeometric differential equation and the solution of radial displacement in the dimensionless form can be written as

$U(R)=A \bar{W}(R)+B \bar{M}(R)+\omega^{2} \bar{F}(R)$,

$\bar{W}(R)=e^{(-x / 2)} x^{(-1 / 2)} W_{i, j}(x), \quad$ for $\beta<0$,

$\bar{W}(R)=e^{(x / 2)} x^{(-1 / 2)} W_{i, j}(x), \quad$ for $\beta>0$,

$\bar{M}(R)=e^{(-x / 2)} x^{(-1 / 2)} M_{i, j}(x), \quad$ for $\beta<0$,

$\bar{M}(R)=e^{(x / 2)} x^{(-1 / 2)} M_{i, j}(x), \quad$ for $\beta>0$,

$\bar{F}(R)=\bar{W}(R) \int F(R) M_{i, j}(x) \mathrm{d} R-\bar{M}(R) \int F(R) W_{i, j}(x) \mathrm{d} R$,

$F(R)=\frac{\Omega^{2} e^{-(n-m)(R-\bar{a})} R^{2} e^{(x / 2)} x^{(1 / 2)}}{(1+\nu) M_{i+1, j}(x) W_{i, j}(x)+M_{i, j}(x) W_{i+1, j}(x)}, \quad$ for $\beta<0$,

$F(R)=\frac{\Omega^{2} e^{-(n-m)(R-\bar{a})} R^{2} e^{(-x / 2)} x^{(1 / 2)}}{(2-\nu) M_{i+1, j}(x) W_{i, j}(x)+M_{i, j}(x) W_{i+1, j}(x)}, \quad$ for $\beta>0$,

where coefficients $A$ and $B$ are arbitrary constants that can be determined from boundary conditions at inner and outer surfaces. $M_{i, j}(x)$ and $W_{i, j}(x)$ are Whittaker's functions presented by Abramowitz and Stegun [1].

$$
\begin{aligned}
& M_{i, j}(x)=e^{(-x / 2)} x^{(j+1 / 2)} M\left(\frac{j-i+1}{2}, 1+2 j, x\right), \\
& W_{i, j}(x)=e^{(-x / 2)} x^{(j+1 / 2)} U\left(\frac{j-i+1}{2}, 1+2 j, x\right),
\end{aligned}
$$

where $M(j-i+1 / 2,1+2 j, x)$ and $U(j-i+1 / 2,1+2 j, x)$ are the hypergeometric and Kummer functions with $i=(\nu-1 / 2), j=1$, and $x=-\beta b R$ for $\beta<0$ and $i=(1 / 2-\nu)$, $j=1$, and $x=\beta b R$ for $\beta>0$, respectively. The Whittaker's functions $M_{i, j}(x)$ and $W_{i, j}(x)$ converge for $|x|<1$. Substituting equation (12) into equations (7)-(9) and letting 


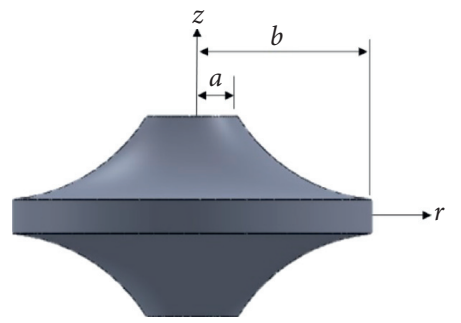

(a)

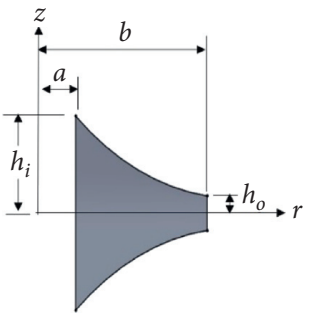

(b)

Figure 1: Annular FGM disk with variable thickness.

$\left(\Sigma_{r}, \Sigma_{\theta}\right)=\left(\left(1-v^{2}\right) / E_{i} \cdot\left(\sigma_{r}, \sigma_{\theta}\right)\right)$, the radial and circumferential stresses in dimensionless forms are

$$
\begin{aligned}
\Sigma_{r}(R)= & A \Sigma_{r_{\bar{W}}}(R)+B \Sigma_{r_{\bar{M}}}(R)+\Sigma_{r_{\bar{F}}}(R) \omega^{2}, \\
\Sigma_{\theta}(R)= & A \Sigma_{\theta_{\bar{W}}}(R)+B \Sigma_{\theta_{\bar{M}}}(R)+\Sigma_{\theta_{\bar{F}}}(R) \omega^{2}, \\
\Sigma_{r_{\bar{W}}}(R)=-e^{-m(R-\bar{a})} R^{-1} e^{(-x / 2)} x^{(-1 / 2)} W_{i+1, j}(x), & \text { for } \beta<0, \\
\Sigma_{r_{\bar{W}}}(R)= & e^{-m(R-\bar{a})} R^{-1} e^{(x / 2)} x^{(-1 / 2)} \\
& \cdot\left[(x-1+2 v) W_{i, j}(x)-W_{i+1, j}(x)\right],
\end{aligned}
$$

$\Sigma_{r_{\bar{M}}}(R)=(1+v) e^{-m(R-\bar{a})} R^{-1} e^{(-x / 2)} x^{(-1 / 2)} M_{i+1, j}(x), \quad$ for $\beta<0$,

$$
\begin{aligned}
\sum_{r_{\bar{M}}}(R)= & e^{-m(R-\bar{a})} R^{-1} e^{(x / 2)} x^{(-1 / 2)} \\
& \cdot\left[(x-1+2 v) M_{i, j}(x)+(2-v) M_{i+1, j}(x)\right], \text { for } \beta>0,
\end{aligned}
$$

$$
\Sigma_{r_{\bar{F}}}(R)=\Sigma_{r_{\bar{W}}}(R) \int F(R) M_{i, j}(x) \mathrm{d} R-\Sigma_{r_{\bar{M}}}(R) \int F(R) W_{i, j}(x) \mathrm{d} R,
$$

$$
\Sigma_{\theta_{\bar{W}}}(R)=\left(1-v^{2}\right) e^{-m(R-\bar{a})} R^{-1} \bar{W}(R)+v \Sigma_{r_{\bar{W}}}(R),
$$

$\Sigma_{\theta_{\bar{M}}}(R)=\left(1-v^{2}\right) e^{-m(R-\bar{a})} R^{-1} \bar{M}(R)+\nu \Sigma_{r_{\bar{M}}}(R)$,

$$
\Sigma_{\theta_{\bar{F}}}(R)=\left(1-\nu^{2}\right) e^{-m(R-\bar{a})} R^{-1} \bar{F}(R)+\nu \Sigma_{r_{\bar{F}}}(R) .
$$

At the inner and outer surfaces, there are various boundary conditions. We investigate the following cases to find elastic solutions for the rotating FEM annular disk under different boundary conditions. The annular disk rotates at constant angular velocity $\omega$.

2.1. Both Edges in Inner and Outer Surfaces Are Subjected to Pressure. In this case, the inner surface is subjected to internal pressure $P_{i}$ and the outer surface is subjected to external pressure $P_{o}$, i.e., $\sigma_{r}(a)=-P_{i}$ and $\sigma_{r}(b)=-P_{o}$. Then,

$$
\begin{aligned}
& A=A_{P_{i}} P_{i}+A_{P_{0}} P_{0}+A_{\omega} \omega^{2}, \\
& B=B_{P_{i}} P_{i}+B_{P_{0}} P_{0}+B_{\omega} \omega^{2},
\end{aligned}
$$

$A_{P_{i}}=-\frac{\left(1-v^{2}\right) D_{22}}{E_{i}\left(D_{11} D_{22}-D_{12} D_{21}\right)}$,

$A_{P_{0}}=\frac{\left(1-v^{2}\right) D_{12}}{E_{i}\left(D_{11} D_{22}-D_{12} D_{21}\right)}$,

$$
A_{\omega}=\frac{-D_{13} D_{22}+D_{12} D_{23}}{D_{11} D_{22}-D_{12} D_{21}},
$$$$
B_{P_{i}}=\frac{\left(1-v^{2}\right) D_{21}}{E_{i}\left(D_{11} D_{22}-D_{12} D_{21}\right)},
$$$$
B_{P_{0}}=-\frac{\left(1-v^{2}\right) D_{11}}{E_{i}\left(D_{11} D_{22}-D_{12} D_{21}\right)} \text {, }
$$$$
B_{\omega}=\frac{-D_{11} D_{23}+D_{13} D_{21}}{D_{11} D_{22}-D_{12} D_{21}},
$$

$$
\begin{aligned}
& D_{11}=\left.\sum_{r_{\bar{W}}}\right|_{R=\bar{a}}, \\
& D_{12}=\left.\sum_{r_{\bar{M}}}\right|_{R=\bar{a}}, \\
& D_{13}=\left.\sum_{r_{\bar{F}}}\right|_{R=\bar{a}},
\end{aligned}
$$

$$
\begin{aligned}
& D_{21}=\left.\sum_{r_{\bar{W}}}\right|_{R=1}, \\
& D_{22}=\left.\sum_{r_{\bar{M}}}\right|_{R=1}, \\
& D_{23}=\left.\sum_{r_{\bar{F}}}\right|_{R=1} .
\end{aligned}
$$

The radial displacement and radial and circumferential stresses in dimensionless forms are 


$$
\begin{aligned}
U(R) & =\left(A_{P_{i}} \bar{W}+B_{P_{i}} \bar{M}\right) P_{i}+\left(A_{P_{0}} \bar{W}+B_{P_{0}} \bar{M}\right) P_{0}+\left(A_{\omega} \bar{W}+B_{\omega} \bar{M}+\bar{F}\right) \omega^{2}, \\
\Sigma_{r}(R) & =\left(A_{P_{i}} \Sigma_{r_{\bar{W}}}+B_{P_{i}} \Sigma_{r_{\bar{M}}}\right) P_{i}+\left(A_{P_{0}} \Sigma_{r_{\bar{W}}}+B_{P_{0}} \Sigma_{r_{\bar{M}}}\right) P_{0}+\left(A_{\omega} \Sigma_{r_{\bar{W}}}+B_{\omega} \Sigma_{r_{\bar{M}}}+\Sigma_{r_{\bar{F}}}\right) \omega^{2}, \\
\Sigma_{\theta}(R) & =\left(A_{P_{i}} \Sigma_{\theta_{\bar{W}}}+B_{P_{i}} \Sigma_{\theta_{\bar{M}}}\right) P_{i}+\left(A_{P_{0}} \Sigma_{\theta_{\bar{W}}}+B_{P_{0}} \Sigma_{\theta_{\bar{M}}}\right) P_{0}+\left(A_{\omega} \Sigma_{\theta_{\bar{W}}}+B_{\omega} \Sigma_{\theta_{\bar{M}}}+\Sigma_{\theta_{\bar{F}}}\right) \omega^{2} .
\end{aligned}
$$

2.2. Both Edges in Inner and Outer Surfaces Are Free of Constraints (FF). In this case, both edges in the inner and outer surfaces are free of constraints, i.e., $\sigma_{r}(a)=0$ and $\sigma_{r}(b)=0$. The radial displacement and radial and circumferential stresses in dimensionless forms are

$$
\begin{aligned}
U(R) & =\left[A_{\omega} \bar{W}(R)+B_{\omega} \bar{M}(R)+\bar{F}(R)\right] \omega^{2}, \\
\Sigma_{r}(R) & =\left[A_{\omega} \Sigma_{r_{\bar{W}}}(R)+B_{\omega} \Sigma_{r_{\bar{M}}}(R)+\Sigma_{r_{\bar{F}}}(R)\right] \omega^{2}, \\
\Sigma_{\theta}(R) & =\left[A_{\omega} \Sigma_{\theta_{\bar{W}}}(R)+B_{\omega} \Sigma_{\theta_{\bar{M}}}(R)+\Sigma_{\theta_{\bar{F}}}(R)\right] \omega^{2} .
\end{aligned}
$$

Coefficients $A_{\omega}$ and $B_{\omega}$ can be obtained from equations (28)-(31).

2.3. Both Edges in Inner and Outer Surfaces Are Clamped (CC). In this case, the displacement at both edges at the inner and outer surfaces are zero, i.e., $u(a)=0$ and $u(b)=0$. The radial displacement and radial and circumferential stresses in dimensionless forms are

$$
\begin{aligned}
U(R) & =\left[A_{\mathrm{CC}} \bar{W}(R)+B_{\mathrm{CC}} \bar{M}(R)+\bar{F}(R)\right] \omega^{2}, \\
\Sigma_{r}(R) & =\left[A_{\mathrm{CC}} \Sigma_{r_{\bar{W}}}(R)+B_{\mathrm{CC}} \Sigma_{r_{\bar{M}}}(R)+\Sigma_{r_{\bar{F}}}(R)\right] \omega^{2}, \\
\Sigma_{\theta}(R) & =\left[A_{\mathrm{CC}} \Sigma_{\theta_{\bar{W}}}(R)+B_{\mathrm{CC}} \Sigma_{\theta_{\bar{M}}}(R)+\Sigma_{\theta_{\bar{F}}}(R)\right] \omega^{2} .
\end{aligned}
$$

Coefficients $A_{\mathrm{CC}}$ and $B_{\mathrm{CC}}$ can be represented as

$$
\begin{aligned}
A_{\mathrm{CC}} & =\frac{-G_{13} G_{22}+G_{12} G_{23}}{G_{11} G_{22}-G_{12} G_{21}}, \\
B_{\mathrm{CC}} & =\frac{-G_{11} G_{23}+G_{13} G_{21}}{G_{11} G_{22}-G_{12} G_{21}}, \\
G_{11} & =\left.\bar{W}(R)\right|_{R=\bar{a}}, \\
G_{12} & =\left.\bar{M}(R)\right|_{R=\bar{a}}, \\
G_{13} & =\left.\bar{F}(R)\right|_{R=\bar{a}}, \\
G_{21} & =\left.\bar{W}(R)\right|_{R=1}, \\
G_{22} & =\left.\bar{M}(R)\right|_{R=1}, \\
G_{23} & =\left.\bar{F}(R)\right|_{R=1} .
\end{aligned}
$$

2.4. The Edge in Inner Surface Is Clamped and in Outer Surface Is Free of Constraints (CF). In this case, the displacement at the inner surface is zero and the edge in the outer surface is free of constraints, i.e., $u(a)=0$ and $\sigma_{r}(b)=0$. The radial displacement and radial and circumferential stresses in dimensionless forms are

$$
\begin{aligned}
U(R) & =\left[A_{\mathrm{CF}} \bar{W}(R)+B_{\mathrm{CF}} \bar{M}(R)+\bar{F}(R)\right] \omega^{2}, \\
\Sigma_{r}(R) & =\left[A_{\mathrm{CF}} \Sigma_{r_{\bar{W}}}(R)+B_{\mathrm{CF}} \Sigma_{r_{\bar{M}}}(R)+\Sigma_{r_{\bar{F}}}(R)\right] \omega^{2}, \\
\Sigma_{\theta}(R) & =\left(A_{\mathrm{CF}} \Sigma_{\theta_{\bar{W}}}(R)+B_{\mathrm{CF}} \Sigma_{\theta_{\bar{M}}}(R)+\Sigma_{\theta_{\bar{F}}}(R) \omega^{2} .\right.
\end{aligned}
$$

Coefficients $A_{\mathrm{CF}}$ and $B_{\mathrm{CF}}$ can be represented as

$$
\begin{gathered}
A_{\mathrm{CF}}=\frac{-G_{13} D_{22}+G_{12} D_{23}}{G_{11} D_{22}-G_{12} D_{21}}, \\
B_{\mathrm{CF}}=\frac{-G_{11} D_{23}+G_{13} D_{21}}{G_{11} D_{22}-G_{12} D_{21}} .
\end{gathered}
$$

Constants in $A_{\mathrm{CF}}$ and $B_{\mathrm{CF}}$ can be obtained from equations (31) and (36).

2.5. The Edge in Inner Surface Is Free of Constraints and in Outer Surface Is Clamped (FC). In this case, the edge in the inner surface is free of constraints and the displacement at the outer surface is zero, i.e., $\sigma_{r}(a)=0$ and $u(b)=0$. The radial displacement and radial and circumferential stresses in dimensionless forms are

$$
\begin{aligned}
& U(R)=\left[A_{\mathrm{FC}} \bar{W}(R)+B_{\mathrm{FC}} \bar{M}(R)+\bar{F}(R)\right] \omega^{2}, \\
& \Sigma_{r}(R)=\left[A_{\mathrm{FC}} \Sigma_{r_{\bar{W}}}(R)+B_{\mathrm{FC}} \Sigma_{r_{\bar{M}}}(R)+\Sigma_{r_{\bar{F}}}(R)\right] \omega^{2}, \\
& \Sigma_{\theta}(R)=\left[A_{\mathrm{FC}} \Sigma_{\theta_{\bar{W}}}(R)+B_{\mathrm{FC}} \Sigma_{\theta_{\bar{M}}}(R)+\Sigma_{\theta_{\bar{F}}}(R)\right] \omega^{2} .
\end{aligned}
$$

Coefficients $A_{\mathrm{FC}}$ and $B_{\mathrm{Fc}}$ can be represented as

$$
\begin{gathered}
A_{\mathrm{FC}}=\frac{-G_{22} D_{13}+G_{23} D_{12}}{G_{22} D_{11}-G_{21} D_{12}}, \\
B_{\mathrm{FC}}=\frac{-G_{23} D_{11}+G_{21} D_{13}}{G_{22} D_{11}-G_{21} D_{12}} .
\end{gathered}
$$

Constants in $A_{\mathrm{FC}}$ and $B_{\mathrm{FC}}$ can be obtained from equations (30) and (37).

\section{Special Cases}

In the limiting case, when $k$ is taken to be zero in equation (1), the thickness of the discussed FEM disk is uniform. When $m$ and $n$ are taken to be zero in equations (3) and (4), the discussed FEM disk is reduced to homogeneous material. From equation (11), the governing equation of radial displacement for the rotating uniform and homogeneous disk in dimension forms becomes

$$
r \frac{\mathrm{d}^{2} u}{\mathrm{~d} r^{2}}+\frac{\mathrm{d} u}{\mathrm{~d} r}-\frac{1}{r} u=-\frac{\left(1-v^{2}\right) \rho_{i} \omega^{2}}{E_{i}} r^{2} .
$$


The radial displacement and radial and circumferential stresses are

$$
\begin{aligned}
u(r) & =c_{1} r+c_{2} \frac{1}{r}-\frac{\left(1-v^{2}\right) \rho_{i} \omega^{2}}{8 E_{i}} r^{3}, \\
\sigma_{r}(r) & =\frac{E_{i}}{1-v^{2}}\left[c_{1}(1+v)-c_{2} \frac{1-\nu}{r^{2}}-\frac{(3+v)\left(1-v^{2}\right) \rho_{i} \omega^{2}}{8 E_{i}} r^{2}\right], \\
\sigma_{\theta}(r) & =\frac{E_{i}}{1-v^{2}}\left[c_{1}(1+v)+c_{2} \frac{1-v}{r^{2}}-\frac{(1+3 v)\left(1-v^{2}\right) \rho_{i} \omega^{2}}{8 E_{i}} r^{2}\right] .
\end{aligned}
$$

These are the well-known solutions for the rotating uniform and homogeneous disk presented in a study by Timoshenko and Goodier [1]; coefficients $c_{1}$ and $c_{2}$ are arbitrary constants that can to be determined from different boundary conditions. To compare with the proposed FEM annular disk, five conditions in the above section are also discussed.

3.1. Both Edges in Inner and Outer Surfaces Are Subjected to Pressure. In this case, the inner surface is subjected to the internal pressure $P_{i}$ and the outer surface is subjected to the internal pressure $P_{o}$, i.e., $\sigma_{r}(a)=-P_{i}$ and $\sigma_{r}(b)=-P_{o}$. Then,

$$
\begin{aligned}
& c_{1}=\frac{(3+v)(1-v) \rho_{i}}{8 E_{i}}\left(a^{2}+b^{2}\right) \omega^{2}+\frac{(1-v)}{E_{i}\left(b^{2}-a^{2}\right)}\left(a^{2} P_{i}-b^{2} P_{o}\right), \\
& c_{2}=\frac{(3+v)(1+v) \rho_{i}}{8 E_{i}} a^{2} b^{2} \omega^{2}+\frac{(1+v) a^{2} b^{2}}{E_{i}\left(b^{2}-a^{2}\right)}\left(P_{i}-P_{o}\right) .
\end{aligned}
$$

3.2. Both Edges in Inner and Outer Surfaces Are Free of Constraints (FF). In this case, both edges in the inner and outer surfaces are free of constraints, i.e., $\sigma_{r}(a)=0$ and $\sigma_{r}(b)=0$. Then,

$$
\begin{aligned}
& c_{1}=\frac{(3+v)(1-v) \rho_{i}}{8 E_{i}}\left(a^{2}+b^{2}\right) \omega^{2}, \\
& c_{2}=\frac{(3+v)(1+v) \rho_{i}}{8 E_{i}} a^{2} b^{2} \omega^{2} .
\end{aligned}
$$

3.3. Both Edges in Inner and Outer Surfaces Are Clamped (CC). In this case, the displacement at both edges at the inner and outer surfaces is zero, i.e., $u(a)=0$ and $u(b)=0$. Then,

$$
\begin{aligned}
& c_{1}=\frac{\left(1-v^{2}\right) \rho_{u}}{8 E_{i}}\left(a^{2}+b^{2}\right) \omega^{2}, \\
& c_{2}=-\frac{\left(1-v^{2}\right) \rho_{i}}{8 E_{u}} a^{2} b^{2} \omega^{2} .
\end{aligned}
$$

3.4. The Edge in Inner Surface Is Clamped and in Outer Surface Is Free of Constraints (CF). In this case, the displacement at the inner surface is zero and the edge in the outer surface is free of constraints, i.e., $\sigma_{r}(a)=0$ and $u(b)=0$. Then,

$$
\begin{aligned}
& c_{1}=\frac{\left(1-v^{2}\right) \rho_{i}}{8 E_{i}} \frac{(1-v) a^{4}+(3+v) b^{4}}{(1+\nu) b^{2}+(1-v) a^{2}} \omega^{2}, \\
& c_{2}=\frac{\left(1-v^{2}\right) \rho_{i}}{8 E_{i}} \frac{(1+v) a^{2}-(3+v) b^{2}}{(1+\nu) b^{2}+(1-v) a^{2}} a^{2} b^{2} \omega^{2} .
\end{aligned}
$$

3.5. The Edge in Inner Surface Is Free of Constraints and in Outer Surface Is Clamped (FC). In this case, the edge in the inner surface is free of constraints and the displacement at the outer surface is zero, i.e., $u(a)=0$ and $\sigma_{r}(b)=0$. Then,

$$
\begin{aligned}
& c_{1}=\frac{\left(1-v^{2}\right) \rho_{i}}{8 E_{i}} \frac{\left[(1-v) b^{4}+(3+v) a^{4}\right]}{\left[(1+v) a^{2}+(1-v) b^{2}\right]} \omega^{2}, \\
& c_{2}=\frac{\left(1-v^{2}\right) \rho_{i}}{8 E_{i}} \frac{\left[(1+v) b^{2}-(3+v) a^{2}\right]}{\left[(1+v) a^{2}+(1-v) b^{2}\right]} a^{2} b^{2} \omega^{2} .
\end{aligned}
$$

\section{Numerical Results and Discussion}

To check the correctness of the proposed analytical solutions in this paper, two functionally graded material annular disks are considered in this paper. The inner and outer radii of both disks are $a=0.2 \mathrm{~m}$ and $b=1 \mathrm{~m}$. The thickness and properties of two disks are shown in Tables 1 and 2. The inner surfaces of both FEM annular disks are nickel (Ni), the outer surface of disk 1 is silicon nitride $\left(\mathrm{Si}_{3} \mathrm{~N}_{4}\right)$, and the outer surface of disk2 is zirconia $\left(\mathrm{ZrO}_{2}\right)$. For disk1, $\beta<0$, and for disk2, $\beta>0$. Both disks rotate at constant angular velocity $\omega=150(\mathrm{rad} / \mathrm{sec})$, and the inner and outer surfaces are subjected to pressure $P_{i}=100 \mathrm{MPa}$ and $P_{o}=10 \mathrm{MPa}$, respectively.

For the sake of completeness, comparisons between homogeneous disks and two FGM annular disks are shown in Figures 2 and 3. Note that, for two FGM annular disks considered herein, the convergence condition of Whittaker's functions, $|x|<1$, is satisfied [9]. Observing the distributions of the displacement and stresses between the homogeneous disk and FGM disk, the FGM disk has better mechanical properties than the homogeneous disk.

Figures 4 and 5 show that displacement and stresses of FEM annular disk1 are subjected to $P_{i}$ and $P_{o}$, respectively. In Figure 4, the inner surface of FEM annular disk1 is subjected to internal pressure $P_{i}$ and there is no pressure applied on the outer surface. Radial stress is in compression state and circumferential stress is in tension state. Radial displacement and radial and circumferential stresses all decrease with the increase of $R$. The maximums of radial displacement and radial and circumferential stresses are all at the inner surface. In Figure 5, the outer surface of FEM annular disk 1 is subjected to external pressure $P_{o}$ and there is no pressure applied on the inner surface. Radial and 
TABLE 1: Thickness and properties of disk1.

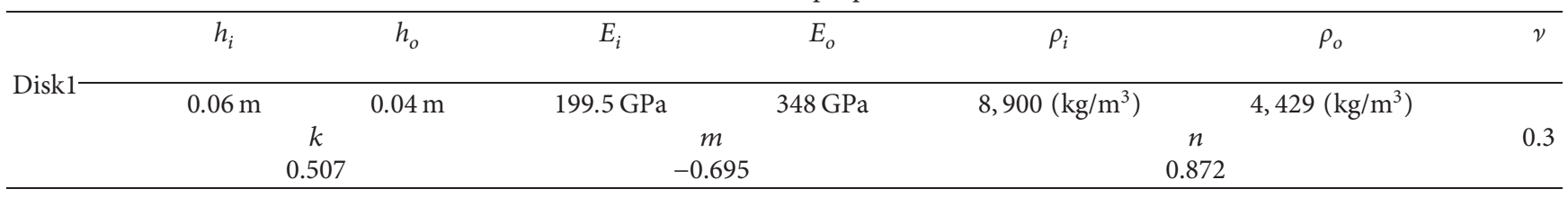

TABle 2: Thickness and properties of disk2.

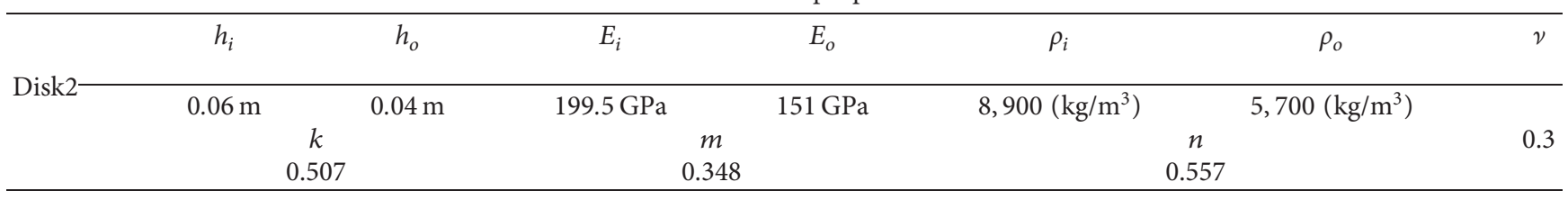

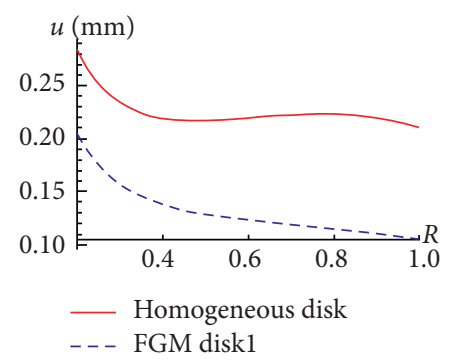

(a)

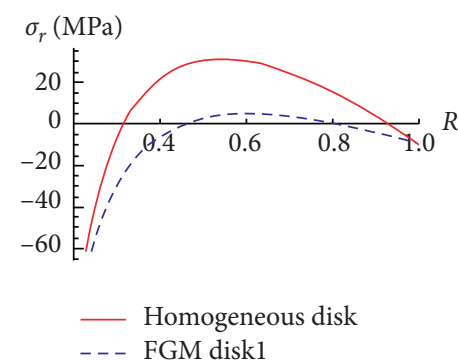

(b)

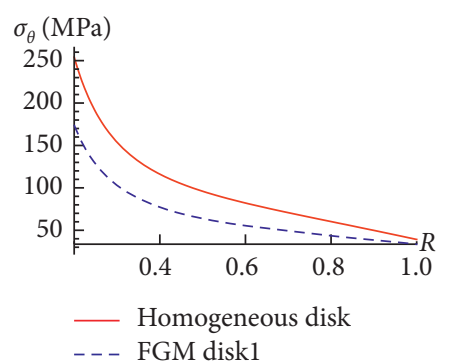

(c)

FIGURE 2: Comparisons between uniform and homogeneous disk and FGM disk1. (a) Radial displacement $u$. (b) Radial stress $\sigma_{r}$. (c) Circumferential stress $\sigma_{\theta}$.

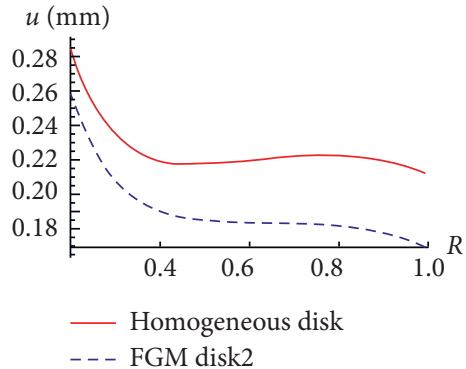

(a)

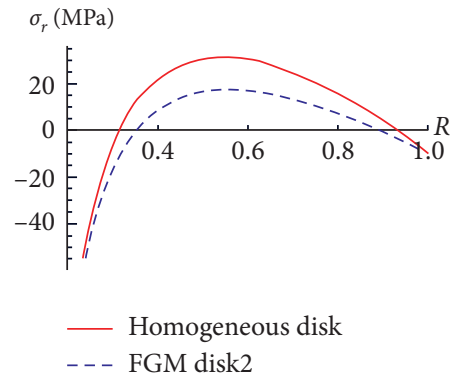

(b)

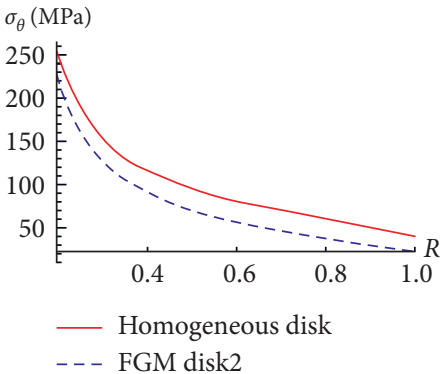

(c)

FIGURE 3: Comparisons between uniform and homogeneous disk and FGM disk2. (a) Radial displacement $u$. (b) Radial stress $\sigma_{r}$.

(c) Circumferential stress $\sigma_{\theta}$.

circumferential stresses are both in compression. The maximum of radial stress is at the outer surface, and compressive stress increases with the increase of $R$. The radial displacement and circumferential stress, from the inner surface to the outer surface, both first increase and then decrease. The maximum of radial displacement occurs around $R=0.27$ and the maximum of circumferential stress occurs around $R=0.43$. Distributions of the displacement and stresses for FEM annular disk2 subjected to pressures at the inner and outer surfaces are like FEM annular disk1.
Figures 6 and 7 depict the displacement and stresses for the two rotating FEM annular disks under various boundary conditions at their inner and outer surfaces. In these two figures, we can find the radial displacement $u(r)$ and circumferential stress $\sigma_{\theta}(r)$ under FF boundary condition are larger throughout the disks. Another radial displacement $u(r)$ under CC boundary condition and radial stress $\sigma_{r}(r)$ under FC boundary condition are smaller throughout the disks. Figures 6 and 7 also show that radial stress $\sigma_{r}(r)$ under $\mathrm{CF}$ and $\mathrm{CC}$ boundary conditions and circumferential stress 


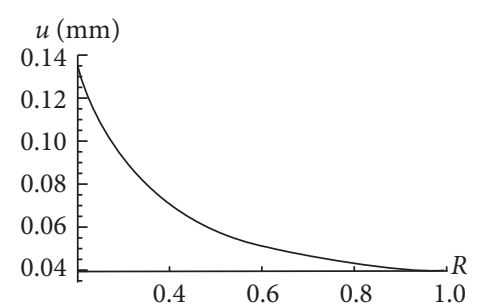

(a)

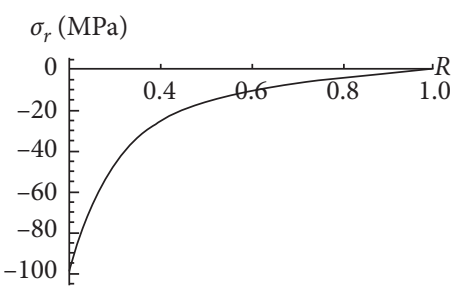

(b)

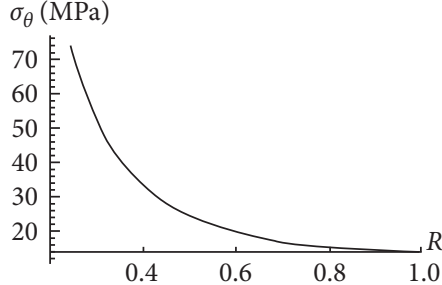

(c)

FIgURE 4: FEM annular disk1 subjected to internal pressure $P_{i}$ only. (a) Radial displacement $u$. (b) Radial stress $\sigma_{r}$. (c) Circumferential stress $\sigma_{\theta}$.

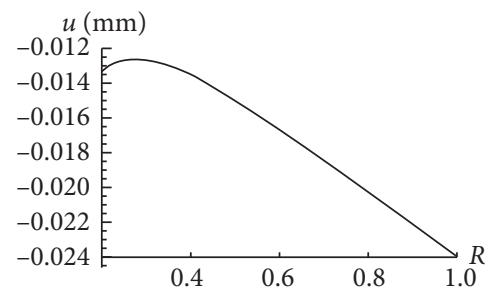

(a)

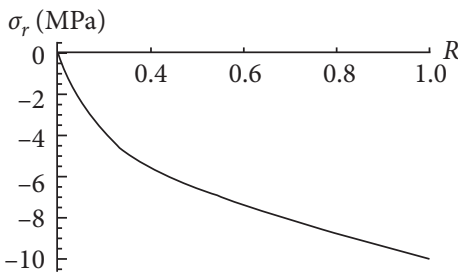

(b)

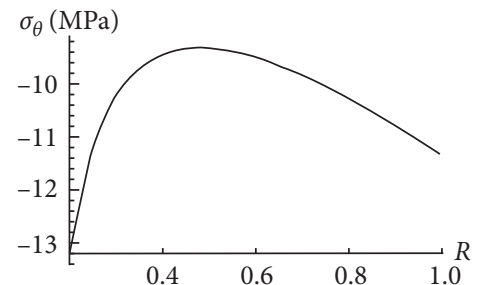

(c)

Figure 5: FEM annular disk1 subjected to external pressure $P_{o}$ only. (a) Radial displacement $u$. (b) Radial stress $\sigma_{r}$. (c) Circumferential stress $\sigma_{\theta}$.

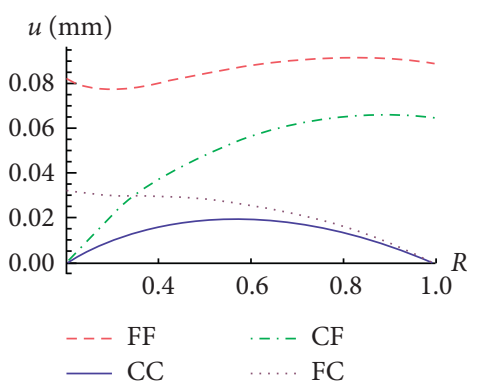

(a)

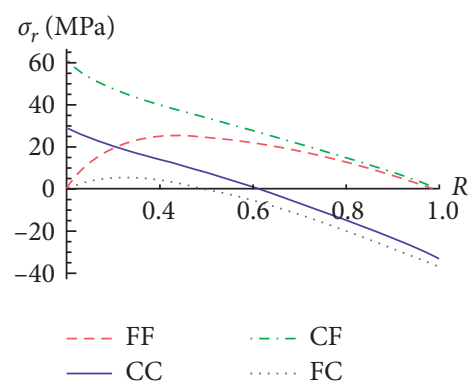

(b)

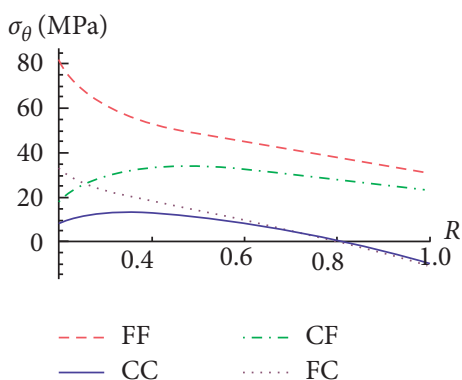

(c)

Figure 6: Radial displacement and radial and circumferential stresses for FEM disk1 under various boundary conditions. (a) Radial displacement $u$. (b) Radial stress $\sigma_{r}$. (c) Circumferential stress $\sigma_{\theta}$.

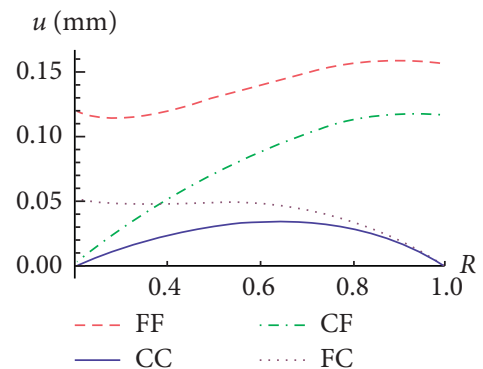

(a)

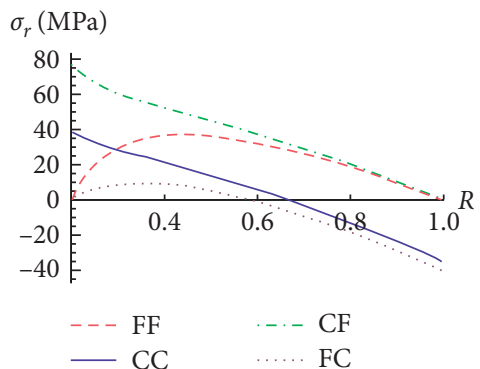

(b)

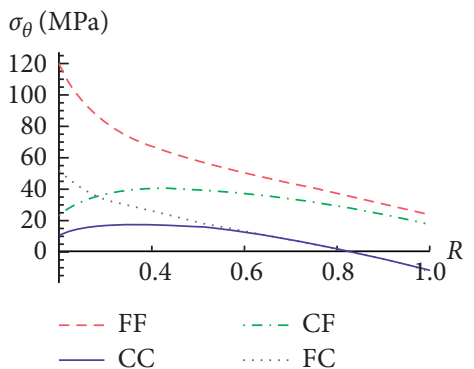

(c)

Figure 7: Radial displacement and radial and circumferential stresses for FEM disk2 under various boundary conditions. (a) Radial displacement $u$. (b) Radial stress $\sigma_{r}$. (c) Circumferential stress $\sigma_{\theta}$. 
$\sigma_{\theta}(r)$ under FF and FC boundary conditions; both decrease with the increase of $R$; the largest and smallest values are at the inner and outer surfaces, respectively. The circumferential stress $\sigma_{\theta}(r)$ under FC boundary condition is larger than that under CC boundary condition for $R \leq 0.8$, and this situation is reversed for $R>0.8$.

\section{Conclusions}

In this paper, closed-form solutions are proposed for mechanical behaviours of the rotating FGM annular disk. The material properties and thickness of the discussed FGM annular disk are assumed to vary radially in exponentially-varying functions. Based on the infinitesimal theory of elasticity, the derived governing equation of radial displacement is a generalized confluent hypergeometric differential equation and the solution is represented by Whittaker's functions. For different FGM materials considered herein, the convergent condition of Whittaker's functions is satisfied. The displacement and stresses are evaluated when the FGM annular disk is subjected to internal and external pressures at the inner and outer surfaces. Furthermore, applying to general cases, four boundary conditions are also investigated. They are FF (both edges in the inner and outer surfaces are free of constraints), CC (both edges in the inner and outer surfaces are clamped), CF (inner edge is clamped, outer edge is free of constraints), and FC (inner edge is free of constraints, outer edge is clamped).

Diagrams of displacement and stresses versus dimensionless radius are plotted to compare mechanical behaviours of the traditionally homogeneous disk with FGM disk. In addition, the response of the proposed FEM annular disk subjected to internal pressure at the inner surface, external pressure at the outer surface and four general boundary conditions are plotted, respectively. The following results are the summaries of the most important achievements of the paper.

(1) The radial displacement and stresses of the proposed FGM disk are smaller than the traditionally homogeneous disk.

(2) When the proposed FGM disk is subjected to internal pressure only, radial stress is in compression state and circumferential stress is in tension state. Radial displacement and radial and circumferential stresses all decrease with the increase of the radius. The maximums of radial displacement and radial and circumferential stresses are all at the inner surface.

(3) When the proposed FGM disk is subjected to external pressure only, radial and circumferential stresses are both in compression. The radial compression stress increases with the increase of radius, and the maximum of radial stress is at the outer surface. Both the radial displacement and circumferential stress first increase and then decrease from inner to outer surface.

(4) Considering the proposed FGM disk under FF, CC, $\mathrm{CF}$, and FF boundary conditions, radial displacement and circumferential stress under FF boundary conditions are larger throughout the disks. Another radial displacement under CC boundary condition and radial stress under FC boundary condition are smaller throughout the disks. Radial stress under CF and CC boundary conditions and circumferential stress under FF and FC boundary conditions both decrease with the increase of radius, the largest and smallest values are at the inner and outer surfaces, respectively.

The presented analytical solutions of FGM stress analysis are very helpful in the predesign stage of engineering applications. Particularly, it can be applied to shrink-fitted assemblies and sandwich structures with functionally graded materials. Other factors should be considered more carefully in future research, such as the thermal resource, hydrostatic pressure, or magnetic force. In the future, we may extend this research to explore microtubules and micro/nano electromechanical systems.

\section{Data Availability}

The data of thickness and properties used to support the findings of this study are included within Tables 1 and 2 .

\section{Conflicts of Interest}

The author declares that there are no conflicts of interest regarding the publication of this paper.

\section{References}

[1] S. P. Timoshenko and J. N. Goodier, Theory of Elasticity, McGraw-Hill, New York, NY, USA, 3rd edition, 1970.

[2] P. Nayak and K. Saha, "Elastic limit angular speed of solid and annular disks under thermomechanical loading," International Journal of Engineering, Science and Technology, vol. 8, no. 2, pp. 30-45, 2016.

[3] V. Yildirim, "Closed-form formulas for hyperbolically tapered rotating disks made of traditional materials under combined thermal and mechanical loads," International Journal of Engineering and Applied Sciences, vol. 10, no. 2, pp. 73-92, 2018.

[4] J. F. Durodola and O. Attia, "Deformation and stresses in functionally graded rotating disks," Composites Science and Technology, vol. 60, no. 7, pp. 987-995, 2000.

[5] Z. Shi, T. Zhang, and H. Xiang, "Exact solutions of heterogeneous elastic hollow cylinders," CompositeStructures, vol. 79, no. 1, pp. 140-147, 2007.

[6] L. H. You, J. X. Wang, and B. P. Tang, "Deformations and stresses in annular disks made of functionally graded materials subjected to internal and/or external pressure," Meccanica, vol. 44, no. 3, pp. 283-292, 2009.

[7] T. Dai and H.-L. Dai, "Investigation of mechanical behavior for a rotating FGM circular disk with a variable angular speed," Journal of Mechanical Science and Technology, vol. 29, no. 9, pp. 3779-3787, 2015.

[8] M. Z. Nejad, M. Abedi, M. Lotfian, and M. Ghannad, "Exact and numerical elastic analysis for the FGM thick-walled cylindrical pressure vessels with exponentially-varying properties," Archives of Metallurgy and Materials, vol. 61, 2016.

[9] M. Abramowitz and I. A. Stegun, Handbook of Mathematical Functions, US Government Printing Office, Washington, DC, USA, 5th edition, 1966. 
[10] M. Gharibi, M. Zamani Nejad, and A. Hadi, "Elastic analysis of functionally graded rotating thick cylindrical pressure vessels with exponentially-varying properties using power series method of Frobenius," Journal of Computational Applied Mechanics, vol. 48, no. 1, pp. 89-98, 2017.

[11] M. H. Sadd, Theory, Applications, and Numerics, Academic Press, Cambridge, MA, USA, 2nd edition, 2009.

[12] A. K. Thawait, "Stress analysis of rotating cylindrical pressure vessel of functionally graded material by element based material gradation," Research \& Reviews: Journal of Physics, vol. 5, no. 3, pp. 7-15, 2018.

[13] A. N. Eraslan and T. Akis, "On the plane strain and plane stress solutions of functionally graded rotating solid shaft and solid disk problems," Acta Mechanica, vol. 181, no. 1-2, pp. 43-63, 2006.

[14] A. M. Zenkour, "On the magneto-thermo-elastic responses of FG annular sandwich disks," International Journal of Engineering Science, vol. 75, pp. 54-66, 2014.

[15] M. R. Akbari and J. Ghanbari, "Analytical exact solution for functionally graded rotating disks under non-symmetric thermal and mechanical loads," Materials Research Express, vol. 6, no. 5, Article ID 056545, 2019.

[16] S. Strashnov, S. Alexandrov, and L. Lang, "Description of residual stress and strain fields in FGM hollow disc subject to external pressure," Materials, vol. 12, no. 3, p. 440, 2019.

[17] M. R. Salehi Kolahi, M. Karamooz, and H. Rahmani, "Elastic analysis of shrink-fitted thick FGM cylinders based on linear plane elasticity theory," Mechanics of Advanced Composite Structures, vol. 7, no. 1, pp. 121-127, 2020.

[18] A. Yildirim, D. Yarimpabuç, and K. Celebi, "Transient thermal stress analysis of functionally graded annular fin with free base," Journal of Thermal Stresses, vol. 43, no. 9, pp. 1138-1149, 2020.

[19] L. H. You, Y. Y. Tang, J. J. Zhang, and C. Y. Zheng, "Numerical analysis of elastic-plastic rotating disks with arbitrary variable thickness and density," International Journal of Solids and Structures, vol. 37, no. 52, pp. 7809-7820, 2000.

[20] M. Bayat, B. B. Sahari, A. M. S. Hamouda, M. Saleem, and E. Mahdi, "On the stress analysis of functionally graded gear wheels with variable thickness," International Journal for Computational Methods in Engineering Science and $\mathrm{Me}$ chanics, vol. 9, no. 2, pp. 121-137, 2008.

[21] M. H. Jalali and B. Shahriari, "Elastic stress analysis of rotating functionally graded annular disk of variable thickness using finite difference method," Mathematical Problems in Engineering, vol. 2018, Article ID 1871674, , 2018.

[22] S. Yildirim and N. Tutuncu, "On the inertio-elastic instability of variable-thickness functionally-graded disks," Mechanics Research Communications, vol. 91, pp. 1-6, 2018.

[23] A. K. Thawait, L. Sondhi, S. Sanyal, and S. Bhowmick, "Stress and deformation analysis of clamped functionally graded rotating disks with variable thickness," Mechanics and Mechanical Engineering, vol. 23, no. 1, pp. 202-211, 2019.

[24] M. Bayat, B. Sahari, M. Saleem, E. Dezvareh, and A. Mohazzab, "Analysis of functionally graded rotating disks with parabolic concave thickness applying an exponential function and the Mori-Tanaka scheme," IOP Conference Series: Materials Science and Engineering, vol. 17, no. 1, Article ID 012005, 2011.

[25] S.-Y. Leu and L.-C. Chien, "Thermoelastic analysis of functionally graded rotating disks with variable thickness involving non-uniform heat source," Journal of Thermal Stresses, vol. 38, no. 4, pp. 415-426, 2015.
[26] Y. Zheng, H. Bahaloo, D. Mousanezhad, E. Mahdi, A. Vaziri, and H. Nayeb-Hashemi, "Stress analysis in functionally graded rotating disks with non-uniform thickness and variable angular velocity," International Journal of Mechanical Sciences, vol. 119, pp. 283-293, 2016.

[27] V. Yildirim, "Thermomechanical characteristics of a functionally graded mounted uniform disc with/without rigid casing," Journal of Aerospace Technology and Management, vol. 11, 2019.

[28] B. Shahriari and M. Safari, "Stress analysis of FGM rotating disk subjected to mechanical and thermal loads in aircraft gas turbine engine," Mechanics of Advanced Composite Structures, vol. 7, no. 1, pp. 1-13, 2020.

[29] Y. Gao, W.-S. Xiao, and H. Zhu, "Nonlinear vibration of functionally graded nanotubes using nonlocal strain gradient theory and a two-steps perturbation method," Structural Engineering and Mechanics, vol. 69, no. 2, pp. 205-219, 2019.

[30] T. Yu, J. Zhang, H. Hu, and T. Q. Bui, “A novel size-dependent quasi-3D isogeometric beam model for two-directional FG microbeams analysis," Composite Structures, vol. 211, pp. 76-88, 2019. 